\title{
Germanica
}

36 | 2005

Le pouvoir de la musique dans l'espace de langue allemande : fascination et suspicion

\section{Le lien entre musique et inconscient dansDas Spielwerk und die Prinzessin de Franz Schreker}

"Das Verhältnis der Musik zum Unbewussten in Das Spielwerk und die

Prinzessin von Franz Schreker ».

\section{Alain Leduc}

\section{OpenEdition}

\section{Journals}

Édition électronique

URL : http://journals.openedition.org/germanica/1513

DOI : 10.4000/germanica. 1513

ISSN : 2107-0784

\section{Éditeur}

Université de Lille

\section{Édition imprimée}

Date de publication : 30 juin 2005

Pagination : 45-65

ISBN : 9782913857155

ISSN : 0984-2632

\section{Référence électronique}

Alain Leduc, « Le lien entre musique et inconscient dansDas Spielwerk und die Prinzessin de Franz Schreker », Germanica [En ligne], 36 | 2005, mis en ligne le 10 juillet 2012, consulté le 06 octobre 2020 URL : http://journals.openedition.org/germanica/1513; DOI : https://doi.org/10.4000/germanica.1513

Ce document a été généré automatiquement le 6 octobre 2020.

(c) Tous droits réservés 


\title{
Le lien entre musique et inconscient dansDas Spielwerk und die Prinzessin de Franz Schreker
}

\author{
«Das Verhältnis der Musik zum Unbewussten in Das Spielwerk und die \\ Prinzessin von Franz Schreker ».
}

\author{
Alain Leduc
}

\section{Contexte}

1 Tous ceux qui se sont un peu intéressés aux opéras de Schreker (1878-1934) le savent: la quasi-totalité de leurs livrets - écrits, rappelons-le, par le compositeur lui-même ${ }^{1}$, tournent autour de la question musicale. Deux d'entre eux, Der ferne Klang [Le Son lointain] (1910) et Christophorus (1927) l'abordent de manière directe en plaçant la problématique de la création musicale au centre de l'intrigue. Quatre autres choisissent une approche plus symboliste ayant pour cadre un Moyen Âge imaginaire; il s'agit de Flammen [Flammes] (1901), Das Spielwerk und die Prinzessin [Le Carillon et la princesse] (1912), Der Schatzgräber [Le Chercheur de trésor] (1918) et Der singende Teufel [Le Diable chantant] (1928). Les trois derniers cités sont dominés par une figure symbolique empruntée au monde musical : la lyre d'Elis dans Der Schatzgräber, l'orgue d'Amandus dans Der singende Teufel et enfin l'étrange carillon de maître Florian dans Das Spielwerk und die Prinzessin.

2 Cette obsession de la question musicale dans l'œuvre schrékérienne est en soi révélatrice $\mathrm{du}$ lieu et du moment historiques où ces opéras ont été composés. On ne peut vraiment la comprendre sans avoir à l'esprit le bouleversement intellectuel qui a marqué la fin de siècle européenne, en particulier dans la capitale autrichienne et notamment dans le monde musical viennois. Ce bouleversement pourrait, de manière très schématique, être comparé à un réveil, à un retour au principe de réalité faisant suite à un siècle de sacralisation de l'art musical. C'est en terre allemande que la musique, sous l'effet conjoint du discours philosophique et de la constitution du 
répertoire classico-romantique, s'est peu à peu érigée en art suprême, l'art métaphysique par excellence, seul capable de révéler l'harmonie secrète de l'univers ou d'atteindre l'essence du monde. Echappant, de par sa nature vibratoire et profondément ambiguë, plus radicalement à la critique que la littérature ou les beaux arts, la musique a bénéficié tout au long du XIX siècle d'une sorte de statut à part, la garantissant des atteintes du monde réel et de l'histoire. Point culminant de cette évolution, le Gesamtkunstwerk wagnérien aboutit, dans Parsifal, à l'assimilation pure et simple de la musique et de la parole révélée, de l'opéra et de l'office religieux.

Ce n'est pourtant pas en Allemagne, mais à Vienne que cette tradition idéaliste de l'art musical trouvera son véritable accomplissement. Certes, depuis la mort de Schubert en 1928, Vienne est resté pour ainsi dire à la traîne du mouvement romantique qui embrasait le reste de l'Europe, se contentant d'un rôle de spectateur. Mais cette déficience sur le plan de la création va être compensée par un développement sans équivalent de la pratique et des institutions musicales, faisant de Vienne, dans la deuxième moitié $\mathrm{du} \mathrm{xIx}^{\mathrm{e}}$ siècle, la référence absolue dans le domaine musical. Contenue dans le programme même de l'idéalisme romantique, la sacralisation de la musique va trouver un prolongement social et institutionnel dans l'historicisme pratiqué avec un zèle tout particulier dans la capitale autrichienne. Nombreux sont ceux qui ont dénoncé cette tendance qui, en faisant des œuvres du passé, en l'occurrence celles du répertoire classico-romantique, des valeurs de référence indépassables, aboutissait à étouffer la moindre tentative ou possibilité d'évolution. Dans son essai Hofmannsthal et son temps, Hermann Broch résume ainsi les méfaits de l'historicisme dans le domaine musical :

En satisfaisant aux devoirs de sa tradition, Vienne confondait l'atmosphère de musée avec la culture et [...] devint son propre musée. Parce que, chose étrange, Haydn et Mozart, Beethoven et Schubert s'étaient trouvés rassemblés sur ce coin de terre, n'y avaient pas été bien traités mais n'en avaient pas moins composé, Vienne s'organisa en institution musicale ${ }^{2}$.

4 À force de sacralisation, les valeurs de la culture ont fini par fonctionner pour ellesmêmes, pour ainsi dire hors de l'histoire, sans se soucier de l'authenticité de leur rapport à la réalité. En musique, cet académisme radical sera, vers la fin du siècle, principalement incarné par deux figures centrales de la vie musicale viennoise : le compositeur Johannes Brahms et son ami et défenseur Eduard Hanslick, le redoutable critique musical de la Neue freie Presse. Véritables gardiens du temple musical, tous deux veilleront jalousement à ce que les traditions soient respectées, tant au niveau des pratiques que des institutions.

5 Mais soulignons tout de suite un paradoxe : les partisans de Brahms, ceux de l'idéal néoclassique ou historiciste, et leurs «ennemis", parmi lesquels on citera principalement Bruckner et Wolf, défenseurs de la «nouvelle musique », autrement dit de l'esthétique wagnérienne, se rejoignent, au fond, sur la conception idéaliste de l'art musical ; celui-ci reste de toute façon au-delà ou en dehors de l'histoire et échappe, en tant qu'objet de culte métaphysique, à l'emprise de la réalité. En témoignent pareillement, même si c'est au travers d'esthétiques fort dissemblables, l'œuvre néoclassique de Brahms comme les cathédrales sonores de Bruckner.

6 Dans sa célèbre Lettre de Lord Chandos (1902), Hofmannsthal raconte comment son héros prend soudain conscience de la rupture qui s'est établie entre le langage et la réalité qu'il est censé désigner. Ayant acquis une sorte d'autonomie abstraite, liée au 
déploiement de la culture idéaliste tout au long du XIX ${ }^{e}$ siècle, le langage ne parvient plus à coller au réel : l'idée ou le concept font désormais écran entre le moi et le monde. C'est une prise de conscience similaire qui se produit en même temps, à Vienne, dans le domaine musical. Elle commence avec Mahler qui, tout en étant l'héritier de la tradition romantique et wagnérienne, va s'employer à rétablir le lien rompu entre l'idéal et le réel. Son esthétique de la citation, de l'ironie et du décalage lui permet de dénoncer la prétention métaphysique de l'art musical, tout en conservant par là même à l'idéal, en tant qu'utopie, une légitimité «en négatif ». Schönberg et ses disciples iront, quant à eux, encore plus loin dans la critique et abandonneront le vieux langage diatonique, indissociable à leurs yeux de la weltanschauung romantique et donc devenu incapable de rendre compte de la réalité moderne.

7 L'œuvre de Schreker participe de cette même conscience critique à l'égard de la tradition romantique et métaphysique dont elle est elle-même l'héritière. Étant principalement compositeur d'opéras et non de musique pure, c'est sur la scène, par l'intermédiaire de livrets qu'il écrit lui-même, que Schreker va donner sa propre vision critique de la métaphysique musicale issue du romantisme, et en particulier de l'œuvre d'art totale wagnérienne. Si son opéra de jeunesse Flammen, qu'on peut lire comme une métaphore du pouvoir de révélation (mais aussi de destruction) de la musique, reste encore dans le giron du maître de Bayreuth, tout change avec Der ferne Klang, où cette fois Schreker choisit d'être son propre librettiste. Extérieurement déjà, si l'on considère l'intrigue pour elle-même, l'œuvre semble être un désaveu de la mission rédemptrice accordée à la musique, et donc au musicien, au XIX siècle. Fritz, le héros de l'opéra, est de toute évidence un musicien "fin de siècle " que sa quête métaphysique - et obsessionnelle - du « son lointain » conduit à rater à la fois sa vie et son œuvre. Mais la remise en cause du statut transcendantal de la musique se lit de façon beaucoup plus radicale dans l'exploration de l'inconscient à laquelle Schreker, sous l'influence des premiers écrits psychanalytiques ${ }^{3}$, se livre dans son opéra. Le son lointain poursuivi en vain par Fritz s'avère n'être qu'une hallucination de sa subjectivité maladive. L'opéra nous dit du même coup que tout se joue dans les profondeurs de la psyché: la harpe éolienne, le chant des sphères, la mélodie du vent dans les arbres ou sur la mer - autant d'images du son lointain qui hantent l'esprit de Fritz - ne sont que des symboles spatialisés de processus inconscients, ces derniers étant la source de ce qu'on nomme plus communément l'inspiration. Comme dans les symphonies mahlériennes, l'origine métaphysique de l'art musical se trouve ainsi confrontée au principe de réalité, réalité historique et existentielle chez Mahler, réalité psychologique chez Schreker. Dans Le Son lointain, on assiste rien moins qu'à la conscientisation des processus psychiques latents qui ont nourri la métaphysique musicale depuis Wackenroder jusqu'à Wagner. Après cette découverte décisive, Schreker va poursuivre son exploration du lien qui unit la musique aux forces de l'inconscient. L'étape suivante est l'opéra Das Spielwerk und die Prinzessin qui va désormais retenir toute notre attention. Nous commencerons par proposer un bref résumé de l'intrigue, pour autant qu'un tel projet soit réalisable, étant donné le symbolisme complexe et souvent obscur du livret. 


\section{Résumé}

\section{Prologue}

8 Quatre hommes, alors qu'ils fabriquent un brancard pour un jeune homme malade et mourant qui a été trouvé dans un fosse à la lisière du village, racontent ce qui s'est passé jusqu'alors :

La princesse avait un jeune amoureux qui jouait du violon - et il jouait si bien qu'il était le seul à pouvoir faire résonner un carillon très particulier. C'est Maître Florian, le père du jeune homme, qui l'avait conçu et fabriqué, pour qu'il joue de la musique pour le bien des hommes. Mais son assistant Wolf avait reconnu qu'il avait trafiqué le mécanisme de sorte qu'il produise non seulement les sons purs d'Apollon mais aussi ceux, lubriques, de Dionysos. La princesse et le jeune homme s'enivraient de la musique du carillon et se lançaient dans des fêtes de plus en plus orgiaques. Maître Florian désespérait.

Liese, son épouse, participait également à ces fêtes, avec son amant, l'ancien assistant de Florian, Wolf. Finalement, Florian ne put plus supporter ce mauvais usage de carillon mais il ne savait pas s'il devait détruire l'œuvre de sa vie ou son fils et l'amour que celui-ci éprouvait pour la princesse. C'est pour cela qu'il chassa le jeune homme loin de chez lui. Depuis ce moment, le carillon est resté muet. Même Florian ne peut plus lui faire produire des sons.

11 La princesse, inconsolable de la perte de son amoureux, est tombée dans la mélancolie. Liese, à cause de Wolf, a sombré dans la déchéance. On l'appelle maintenant « la Lisedu-fossé ». Personne ne fait plus la fête. La princesse, comme tous les autres habitants du village, ne peut ni vivre ni mourir.

\section{Premier acte}

12 Lise se jette aux pieds de Maître Florian pour lui demander la grâce et l'accès au village pour le jeune homme trouvé. C'est leur fils et l'amoureux de la princesse. Mais le Maître reste inflexible. Au lieu de cela, il essaie comme un fou de réparer le carillon.

Ce jour-là, un jeune homme croise la route du garde du château de la princesse. Il a entendu parler de la princesse malade et du carillon. Lorsqu'il joue par hasard une mélodie sur sa flûte, le mécanisme se remet en marche de façon inexplicable. Le garde ne se sent plus de joie et se remet à espérer que la princesse va guérir. Maître Florian est comme délivré. Mais il met le jeune garçon en garde contre la princesse qui n'a utilisé le mécanisme que pour attirer et pervertir les jeunes gens.

La princesse ne veut plus vivre après avoir supplié en vain Maître Florian de lui rendre son bien-aimé. Elle ne veut rien savoir d'un autre jeune homme qui sait comment faire résonner le carillon. Elle ordonne la préparation d'une dernière grande fête extravagante, au sommet de laquelle elle compte se donner la mort par le feu et y brûler l'instrument. Pour cela, elle convoque Wolf, qui doit déclencher l'incendie. Celuici lui objecte que le carillon, qu'il a contribué a construire, est également une partie de lui-même, argument que la princesse ne veut pas entendre. Lorsqu'elle lui promet de se donner à lui au point culminant de cette orgie, Wolf accepte le marché... [Liese entre alors en scène et oblige la princesse à la suivre jusqu'au chevet de son fils mourant.] 


\section{Deuxième acte} de Saint-Florian, Bruckner, et à sa quête de la musique absolue, on ne peut pas dire que Das Spielwerk und die Prinzessin soit un Künstleroper, un opéra d'artiste, comme l'était Der ferne Klang. Ce n'est pas la composition qui est au centre de l'œuvre, mais la musique elle-même et son pouvoir de fascination. Ce constat soulève d'emblée une question essentielle : de quelle musique parle-t-on?

Qualifié de sorcier par les habitants de la ville, Florian ne se définit à aucun moment comme musicien ; il est juste l'inventeur d'un instrument par l'intermédiaire duquel il espère capter la musique des sphères afin d'en faire bénéficier l'humanité, variante de l'alchimiste à la recherche de la pierre philosophale. La puissance du carillon 
s'apparente donc à un véritable pouvoir dans la mesure où son chant est conçu à l'origine pour éveiller chez l'auditeur les résonances les plus nobles de son âme et le conduire à la félicité parfaite. Le travail humain et subjectif de composition est escamoté par Schreker au profit du seul pouvoir, atemporel et métaphysique, de la musique. Alors que dans Le Son lointain l'œuvre apparaît très clairement comme le fruit d'une interaction entre le but idéal et le vécu subjectif, c'est comme si désormais la musique était déjà écrite et qu'il suffisait de trouver la formule magique pour la contraindre à s'incarner dans le son. Ce retour à une conception mystique de l'art musical marque sans aucun doute une régression par rapport à la modernité de l'opéra précédent. Il permet toutefois à Schreker de développer une réflexion portant non pas sur le processus de création, mais sur le pouvoir de la musique. Ce faisant, Schreker rejoint la position des romantiques allemands, Wackenroder et Hoffmann en tête, qui ont d'abord mesuré la grandeur de la musique à la puissance de ses effets sur la subjectivité humaine.

Schreker introduit certes une dimension humaine et contingente dans ce symbolisme abstrait : l'instrument ne peut s'éveiller que si un musicien est capable de jouer devant lui une mélodie adéquate, véritable formule magique pouvant seule actionner le mystérieux mécanisme. La musique des sphères a donc besoin de l'homme pour s'incarner. Mais ce trait de réalisme trouve vite sa limite. Le fils de Florian, en effet, n'est, pas plus que le "garçon", héros de l'opéra, un compositeur en bonne et due forme, mais plutôt un musicien amateur auquel seul une sensibilité particulière a permis d'être l'élu capable d'actionner le carillon. Schreker, nous l'avons déjà dit, ne s'intéresse pas au processus de création : il supprime délibérément cette étape séparant la sensibilité propre du musicien et la musique idéale du carillon qu'elle déclenche. On passe sans transition de l'une à l'autre. Rien de terrestre, qu'il s'agisse de travail ou de vécu individuel, ne doit altérer la symbolique métaphysique de l'instrument.

Le projet de maître Florian apparaît donc d'emblée de nature dionysiaque, et non apollinienne, comme on le lit parfois. Ce n'est pas par la maitrise classique de la forme, donnant lieu à un ravissement d'ordre spirituel, que le carillon entend exercer son pouvoir sur l'auditoire, mais par la dissolution momentanée de la frontière entre l'individu et l'univers. Florian l'explique lui-même au garçon :

Ich hatt'einen Sohn, jung und fröhlich wie du -

Die Fiedel strich er wie keiner im Land -

Und mein Spiel da drinnen sang Frühlingslieder.

J'avais un fils, jeune et joyeux comme toi -

Il jouait du violon comme nul autre dans la contrée -

Et mon carillon répandait des chants printaniers.

Le carillon entre en résonance avec l'âme du jeune homme, alors plein de la fougue et de la vitalité propres à la jeunesse, et fait résonner « des chants printaniers » qui euxmêmes sont censés se communiquer à l'âme de l'auditeur.

La métaphore schrékérienne du pouvoir de la musique semble donc reposer sur trois instances: le musicien doté de sa seule sensibilité, le carillon qui transforme cette sensibilité en une musique d'essence dionysiaque, et le public qui succombe à son pouvoir d'envoûtement. Mais une question demeure en suspens: si ce n'est pas l'intellect, la maitrise savante de la forme qui explique la fascination qu'exerce l'instrument, de quelle propriété naturelle de la musique celle-ci peut-elle bien se nourrir? S'agit-il du rythme, élément dionysiaque par excellence? Celui des 
bacchanales qui met les foules en transe, comme au début de Tannhäuser ou, mieux, dans Le Sacre du printemps? Les trois passages de l'opéra où Schreker fait entendre le carillon ainsi que les différentes évocations de l'instrument au cours de l'opéra plaident pour une autre dimension, celle du son [Klang]. Dans le préambule qu'il a écrit pour soidisant faciliter la compréhension de son opéra, Schreker qualifie le carillon de "Resonanzwerk ", instrument fondé sur la résonance. Dans les passages où le carillon s'anime au son de la flûte du garçon, mélodie et harmonie se mettent pareillement au service de la pure fascination sonore: les motifs certes existent, mais ils sont littéralement emportés, voire dissous dans le flot tourbillonnant des timbres et des harmoniques. L'orchestre schrékérien donne ici libre cours à sa propre tendance naturelle, à cette esthétique du son qui dominera chez lui jusqu'à Irrelohe et qui culminera dans son chef d'œuvre Die Gezeichneten [Les Stigmatisés].

Le compositeur s'est lui-même à mainte reprise expliqué sur ce trait si caractéristique de sa musique. Le son est pour lui la chair même de la musique, sa réalité la plus profonde et la plus mystérieuse ; c'est l'élément vibratoire de la musique, celui qui, par nature, échappe, du moins partiellement, à la volonté organisatrice du compositeur. Il est au fond tout ce qui déborde de la note, celle-ci étant une entité fixée dans sa hauteur, sa longueur, sa nuance ou son timbre, par le code culturel. En tant que tel, il se manifeste dans la résonance, dans l'épaisseur harmonique du tissu musical, dans cette zone intermédiaire entre la culture, qui le fixe sous forme de note, et la nature où il redevient bruit, puis silence.

La fille du compositeur, Haidy Schreker-Bures, se souvient de la manière dont son père explicitait sa conception si caractéristique du son :

Des sons, y a-t-il un mot plus mal employé, plus méprisé que celui-là, rien qu'un son, de simples sons. Si les grincheux savaient tout ce qu'un son, tout ce qu'un accord peut receler de possibilités expressives, de magie sonore inouie. Déjà petit garçon, j'adorais frapper au piano un de ces accords wagnériens et l'écouter s'éteindre, perdu dans ma rêverie. Il me venait alors d'étranges visions, des images incandescentes issues du monde enchanté de la musique, et une profonde nostalgie. Ce son pur, sans ajout motivique, est, quand on l'utilise avec précaution, un des moyens d'expressions les plus essentiels en musique ${ }^{5}$.

29 À nouveau, la conception "résonante » schrékérienne peut apparaître comme l'héritière de la métaphysique romantique: l'univers entier résonne d'une musique secrète et l'homme n'a pas de mission plus élevée que de parvenir à déchiffrer ce langage fait de vibrations. "La nature est une harpe éolienne, écrit Novalis, un instrument musical dont les notes ne sont que les touches actionnant des cordes plus sublimes en nous ${ }^{6} . "$ Jean Paul ne dit pas autre chose dans un passage d'Hesperus: "Alors, animée par un souffle venu d'en haut, la harpe éolienne de la Création se mit à vibrer et à retentir, et mon âme était une corde sur cette lyre ${ }^{7}$.» L'âme humaine est originellement en sympathie avec la nature. Il faut juste trouver la clef ou la formule pour que la vibration intérieure de l'âme puisse se mettre au diapason de la musique de l'univers. Étant l'art vibratoire et résonant par excellence, il va de soi que l'art musical occupe une place privilégiée dans ce topos romantique : d'essence plus physique et plus spontanément résonante que la poésie, il se pose comme le médiateur idéal entre l'âme et le monde. L'image récurrente de la harpe éolienne (qui apparaît déjà dans Le Son lointain) montre sans ambiguité que la part du travail et de la subjectivité humaine est passée sous silence. L'homme se contente de construire l'instrument (comme Florian) et c'est ensuite le vent qui en joue. Dans cette conception néo-pythagoricienne, la 
musique ne peut être que spontanée, jaillissante, suivant les lois internes de la nature. Le musicien n'est, dans cette perspective, qu'un fidèle transcripteur, ce qui correspond bien au symbolisme du carillon : il n'y a pas de transition « savante » entre la musique réalisée et la sensibilité du musicien, dont l'âme n'est elle-même que le clavier sur lequel vient jouer "la musique du monde». Si on ajoute que le public est aussi en sympathie résonante avec l'instrument, on retrouve la pensée romantique qui veut que tout ce qui vit, homme et nature, baigne dans le même flot vibratoire. Les trois instances qui constituent la métaphore schékérienne du pouvoir de la musique, à savoir musicien/carillon/auditeur, fonctionnent en définitive comme autant de caisses de résonance : l'âme du musicien résonne à la musique du monde qui l'entoure, le carillon entre alors à son tour en résonance et la vibration se communique pour finir à l'âme de celui qui écoute. La dimension humaine, existentielle, liée au travail de composition, est évacuée du schéma symbolique.

\section{Le son et la pulsion}

En sacrifiant à cette conception spontanéiste de la création musicale, il est clair que Florian se privait sans le savoir du garde-fou que constitue la forme apollinienne. En effet, si seule la sensibilité détermine la musique, il faut s'attendre à ce que cette dernière s'écarte bien vite de la voie lumineuse que le maître avait tracée pour elle. La sensibilité est, pour l'essentiel, une donnée originelle de la personnalité ; elle s'enracine au plus profond de la psyché humaine et échappe au contrôle de la raison et de la culture (du « surmoi » pour parler avec Freud). Comme tout ce qui tapisse l'inconscient, elle n'attend souvent qu'une occasion, qu'un stimulus extérieur (événement, rencontre, découverte) pour se manifester. Et c'est ce qui se produit avec le fils de maître Florian. Sa sensibilité proprement musicale qui lui permet, dans un premier temps, de faire résonner des chants printaniers connaît un profond bouleversement lors de la rencontre avec la princesse. C'est à nouveau Florian qui parle :

Da sie ihn ersah, war's um ihn geschehn.

Ein Leben hub an voll der tollsten Laster.

In Nächten verprassten sie ihre Jugend -

Und spielen sollte er immerzu.

Ein Klang schlich sich ein, Bursch, ein frevles Getön

Und drang hinaus über Land und Gau

Verrucht und berauschend, alles verwirrend,

Sinnlos verstrickend zu wildem Reigen.

Quand elle le vit, c'en était fait de lui.

Ils tombèrent dans la plus folle dépravation.

Leur jeunesse se consuma en nuits de débauche -

Et il ne devait plus s'arrêter de jouer.

Un son s'immisça dans son jeu, une infâme vibration,

Et se répandit dans toute la contrée,

Impie et enivrant, brouillant tous les esprits,

Entraînant chacun dans une ronde démente.

31 L'élan vital de la jeunesse se voit pris en charge par la pulsion érotique. Les deux jeunes gens s'abandonnent à une sensualité débridée qui ne laisse que peu de place à l'élément moral et spirituel du sentiment amoureux. Les corps s'attirent sans que les âmes se reconnaissent et la musique du carillon, qui traduit ce nouvel état de la sensibilité du jeune « violoneux », se fait voluptueuse et dionysiaque (au sens orgiaque du terme). Le 
pouvoir de l'instrument s'exprime alors dans toute sa violence, entraînant les habitants de la ville dans la débauche.

Si la conception schrékérienne s'inscrivait entièrement dans la tradition métaphysique de l'art musical, le carillon, incarnation de la musique idéale, resterait en soi exempt de toute responsabilité dans les catastrophes liées à son retentissement ; celles-ci seraient le seul fait d'une déficience morale ou spirituelle dans l'âme du musicien-transcripteur. Le fils de maître Florian comme le garçon se seraient détournés de la pure contemplation esthétique pour céder à une pulsion libidinale et régressive en tombant amoureux de la princesse. Or, plusieurs passages du livret viennent contredire cette hypothèse. Pour commencer, si Florian est bien le porte-parole de la pensée métaphysique pour qui musique est synonyme de sublime, d'élévation et de dépassement, on apprend, à la fin de l'acte 1, qu'il n'a pas travaillé seul à la réalisation du carillon: Wolf, son ancien assistant, avoue à la princesse que l'instrument est en partie son œuvre et qu'il a «un peu saboté » le travail du maître [«Und hab'ihm ein wenig hineingepfuscht... »] Le caractère brutal et primaire de Wolf, dont le nom déjà exprime l'animalité, est l'exacte antithèse de la spiritualité lumineuse recherchée par Florian. Il y a donc à la base même de la conception du carillon un vice de forme que Florian lui-même finira par admettre, au second acte, alors qu'on lui ramène son fils décédé :

Ich hab'was versehn - es stimmt nicht ganz!

Lang'hab'ich gegrübelt - es liegt an dem Werk !

Dort eine Pfeife und da ein paar Saiten -

Auch mit den Glöckchen will sich's nicht reimen.

Was wie Frühling ist und wie Frühling blüht,

Das will eben auch seinen Sommer haben.

J'ai commis une erreur - quelque chose ne va pas!

J'ai longtemps cherché - cela vient du carillon!

Ici un tuyau, là quelques cordes -

Même les clochettes ne s'harmonisent pas.

Ce qui est comme le printemps et comme lui s'épanouit,

Veut aussi avoir son été.

Pourquoi avoir précisé cela, sinon pour signifier que la musique est, dans son essence, un art ambigu. Elle ne se tient pas que du côté de la contemplation et de la poésie, comme l'a naïvement souhaité Florian; en elle s'exprime aussi, originellement, la pulsion désirante - l'élan printanier dont parle Florian - qui est le moteur de toute vie, de toute action humaine. Le principe «Florian» et le principe «Wolf» sont en fait indissolublement liés l'un à l'autre, comme le jour et la nuit. Un passage de la préface rédigée par Schreker pour la partition de l'opéra est on ne peut plus explicite :

«Le Maître» ou «Dieu» ou «la Nature» ont placé dans le cœur de l'homme quelque chose de mystérieux, comme une étrange vibration, un son pour ainsi dire, qui se manifeste en harmonies spectrales pour peu qu'il soit éveillé par la mélodie d'un désir. Mais le carillon de maître Florian, formé de fines membranes, de cordes, de clochettes et de tuyaux, ne sommeille pas que dans le cœur de l'homme, dans l'attente du réveil ; au plus profond de la terre aussi il palpite, il vibre, il aspire à l'appel du printemps, à la mélodie brûlante de l'été et du soleil. [...] Appelez cela la " pulsion » que le Maître a placée dans tout ce qui vit, le miracle de la création dans son éternel renouvellement - ou bien appelez cela "sensualité », " concupiscence perverse »- c'est l'origine de tout le bien et de tout le mal, de la félicité et de la déception, du bonheur et de la misère, de la vie et de la mort : c'est le divin et le diabolique ${ }^{8}$. 
cascription du carillon et l'évocation de l'appel du printemps semblent se rapporter plus ou moins directement aux paroles, citées plus haut, de maître Florian. Si le carillon échappe à son contrôle, c'est parce qu'il est aussi complexe et insondable que l'âme humaine. Il y a pareillement en eux quelque chose de "mystérieux", "vibrant", "retentissant». On retrouve sous la plume de Schreker une sorte de variante de la métaphysique musicale que Schopenhauer expose dans son ouvrage Le Monde comme volonté et représentation. Si la musique est l'art suprême, explique le philosophe, c'est parce qu'elle est, dans sa réalité physique, l'expression même du principe de vie, sans cesse tiraillé entre le désir et la crainte de l'assouvissement. L'homme qui désire agit ; l'homme qui a réalisé son désir s'ennuie et cherche donc de nouveaux objets de désir; et la musique tonale, poursuit Schopenhauer, fonctionne selon le même principe : une mélodie se nourrit de tensions harmoniques tendant vers un but, une résolution. Or, le retour à la tonique, s'il apporte le repos, apporte aussi l'ennui et le désir de repartir vers de nouvelles aventures harmoniques. Il y a certes des musiques plus sages ou plus contemplatives que d'autres, mais aucune n'échappe entièrement à cette bipolarité du désir et de l'ennui. Schopenhauer appelle vouloir-vivre cette énergie qu'il décèle dans l'ensemble de la création, qu'elle soit humaine, animale ou végétale et que Schreker, à sa suite, voit à l'œuvre dans le " sein de la terre ». Le concept romantique de Sehnsucht choisi par le compositeur déploie ici toute la richesse de ses significations; il peut certes s'agir d'une noble aspiration, d'une quête d'idéal, comme dans le cas de maître Florian ; mais la Sehnsucht peut aussi désigner le désir physique de l'être aimé, celui qui anime les différents amants ou courtisans de la princesse.

La vision schrékérienne se sépare de celle de Schopenhauer par la distinction qu'elle opère entre un élément fondamental, présent dès l'origine dans l'âme comme dans la musique, et l'énergie du désir, de la Sehnsucht,qui vient ou non entrer en résonance avec ce fond primitif. Ce qui pose problème dans le parallèle établi par Schopenhauer entre la mélodie et le désir, c'est que tous deux sont déjà des constructions, des réalités organisées et conscientisées ; elle ne rendent pas compte du fond primitif et pulsionnel dont ils sont issus. Cela nous renvoie directement au schéma symbolique bien particulier du carillon: l'instrument mystérieux ne peut être entendu que si une mélodie adéquate parvient à l'éveiller. En termes plus explicites : la mélodie (c'est-àdire la musique en tant que forme) a besoin de la résonance pour exercer son plein effet, de même qu'à l'inverse la résonance originelle ne peut exister qu'en s'incarnant dans une musique (une mélodie) réelle. Schreker s'avère ainsi plus conséquent dans son symbolisme que les métaphysiciens romantiques. Ceux-ci, qu'il s'agisse de Wackenroder, Hoffmann, Novalis ou Schopenhauer, entretiennent constamment la confusion entre la musique comme principe métaphysique et la musique réelle composée par un individu donné. La musique apparaît d'emblée idéale, si bien qu'on se demande s'il y a encore lieu de distinguer entre une symphonie beethovénienne et un air d'opéra italien à la mode. Pour Schreker, si la musique est l'art métaphysique par excellence, c'est uniquement parce qu'elle s'élabore, comme l'âme humaine elle-même, à partir d'un fond vibratoire primitif. Mais encore faut-il que le musicien soit capable de donner un minimum de forme à ce fond: le carillon rappelons-le - n'exerce son pouvoir qu'à travers l'intervention de la Sehnsucht du musicien, sans laquelle le son originel resterait informe et à proprement parler inaudible, de même que la pulsion n'est appelée à prendre forme et, partant, à devenir réelle que dans un individu et un vécu particuliers. 

musique ce que la pulsion [Trieb] est à la psyché humaine. Il est plus originel que le rythme dans l'exacte mesure où la pulsion précède la pulsation, laquelle suppose déjà un minimum d'organisation (la répétition). Il est clair qu'en introduisant ce mot fortement connoté, Schreker se réfère à Freud dont il connaît bien les premiers écrits psychanalytiques. La citation se termine ainsi sur une description de la libido « par delà le bien et le mal » que le père de la psychanalyse n'aurait pas reniée. À ce niveau de profondeur, les énergies de la psyché ne s'encombrent pas de morale ; la pulsion vitale et désirante règne en maitre et il appartiendra à la personnalité de l'individu tout au plus de décider de son orientation future.

Cet aspect moderne de la conception musicale schrékérienne, nul mieux que le musicologue Paul Bekker ne l'a saisi et analysé. Dans son ouvrage Studie zur Kritik der modernen Oper il revient sans cesse sur ce lien élémentaire établi par Schreker entre le Trieb et le Klang pour en montrer toute la force et toute la pertinence :

Le Son lointain, Le Carillon, Les Stigmatisés ne sont pas trois livrets qui se suivent de manière arbitraire, l'auteur ne choisit pas au hasard tel ou tel contenu. Ce sont trois fantaisies sur le même sujet et ce sujet est le son. Il prend la forme symbolique d'un appel mystérieux, de la beauté, de l'élan vital dévastateur, il s'exprime dans la toute puissance des sens, fascinante et enivrante, qui transforme les âmes et les recréée. C'est sous trois aspects différents ce seul et unique sujet qu'on retrouve, cette seule et unique extase créatrice née de l'expérience indescriptible du son, de la manifestation vertigineuse et cosmique de la vibration sonore. C'est elle qui engendre les visions dramatiques de Schreker. Nous voyons donc ici une conception dramatique se développer à partir d'une vision musicale originelle, qui n'est autre que le phénomène typiquement schrékérien du son qui se dissout en harmoniques le véritable élément de transcendance en musique ${ }^{9}$.

Bekker souligne l'ambiguïté fondamentale du son, qui n'est lié à aucun système de valeurs établi, qu'il soit musical ou moral. Le son est l'événement originel et il est en cela aussi mystérieux dans ses résonances que le tréfonds de la psyché humaine. La transcendance dont parle Bekker à la fin du passage n'est plus qu'une transcendance de façade, liée au mystère même, au caractère inconnaissable de la vibration originelle. Le musicien n'y a lui-même accès que dans des moments privilégiés d'inspiration; la vibration se révèle alors à lui sous la forme d'une «vision» qui s'impose à lui, déterminant l'ensemble des paramètres de ses opéras. Bekker met ici le doigt sur ce qu'on pourrait appeler l'axe central tant de la weltanschauung que de l'esthétique de Schreker : toute sa musique, ou presque, sera dédiée à l'exploration des profondeurs désirantes de la psyché humaine. Une citation des écrits théoriques du compositeur revient de manière quasi imparable sous la plume de ses commentateurs et exégètes, elle dit ceci :

J'écris sans schéma préétabli. J'utilise ce qui me vient à l'esprit. Mais - je viens de la musique. Les idées qui me viennent n'ont pas grand-chose de «littéraire ». De mystérieux phénomènes psychiques réclament leur expression musicale. Autour d'eux s'enroule une action extérieure qui, sans que je le veuille, porte d'emblée en elle une forme et une structure musicales ${ }^{10}$.

«Littéraire » est pour Schreker synonyme de construit, de voulu. C'est le domaine de l'intellect. Mais les processus mystérieux de la psyché profonde ne sont d'abord que vibratoires et c'est pourquoi, dans l'inconscient d'un musicien, ils cherchent spontanément à s'exprimer à travers la vibration musicale, dont la forme originelle, 
infiniment complexe et potentiellement riche de toutes les possibilités de l'être, est le son [Klang].

Cela nous renvoie à nouveau directement au symbolisme du carillon : seul le musicien sensible (ou inspiré) est capable de traduire l'inconscient en musique, la pulsion en son; mais cette similitude vibratoire qui permet le passage de l'un à l'autre est une donnée universelle. L'inconscient du musicien, comme celui de n'importe qui, ne s'individualise que dans le vécu; à sa base il est parfaitement indifférencié et collectif, un pur «ça » désirant, dirait Freud. Or, la musique étant, de tous les arts, le seul qui soit en mesure d'entretenir la "résonance originelle » au sein d'une forme intellectuellement organisée, elle possède plus qu'aucun autre le pouvoir d'atteindre et de pénétrer la psyché de l'auditeur. À l'image de Jean-Baptiste Grenouille dans Le Parfum de Patrick Süskind, un musicien qui connaîtrait à fond les lois unissant le son à la pulsion, la musique à l'éros universel, posséderait un pouvoir presque sans limite. Mais le héros de Süskind n'est pas un artiste. Sa science des parfums n'a pas de visée esthétique, mais fonctionnelle. D'autre part, le parfum ou l'odeur font appel à des paramètres beaucoup trop complexes, beaucoup trop "bruts", pour pouvoir être médiatisés dans l'œuvre d'art. Ils ne peuvent s'organiser en mélodie ${ }^{11}$. L'allégorie schrékérienne du carillon pointe en revanche, une trentaine d'années avant le Faustus de Thomas Mann, un danger réel, particulièrement aigu dans le contexte culturel germanique que nous évoquions au début de cet article : celui d'accentuer la donnée primitive ou vibratoire de l'art musical au détriment de sa dimension intellectuelle, de mettre Apollon au service de Dionysos, de soumettre l'esprit à la pulsion. La question musicale qui traverse Le Carillon et la Princesse se retrouve ainsi à l'intersection des angoisses et des interrogations de la fin de siècle européenne, celles-là mêmes qui nourriront bientôt la réflexion freudienne dans Malaise dans la civilisation.

\section{Les enjeux éthiques du Carillon}

Schreker le confesse en toute clarté dans la remarque liminaire qu'il rédige en 1913 pour aider le public à déchiffrer le symbolisme de son opéra :

En effet, cela me rebuterait d'écrire un opéra où la musique n'aurait qu'une fonction de décor ou d'accessoire, ne disposerait plus de la moindre autonomie; et mettre en musique un sujet dont l'idée principale n'engloberait pas toute la musique, que dis-je, ne serait pas la musique même, me rebuterait plus encore ${ }^{12}$.

Mettre la musique au centre de son œuvre lui apparaitt comme quelque chose de naturel et de vital. Mais par «musique » Schreker n'entend pas l'art culturellement établi ; son projet n'est pas d'écrire un Künstleroper comme le Palestrina de Pfitzner. La musique désigne ici l'onde sonore issue de l'inconscient humain. Dans Les Stigmatisés, par exemple, les principaux protagonistes sont une femme peintre et un créateur de spectacles en plein air ; à aucun moment il n'y est question de musiciens, ni même de musique. Dans Le Carillon et la princesse, en revanche, l'effet de mise en abyme est indéniable. Schreker étant musicien et de surcroît son propre librettiste, il est logique que la vision du « son pulsionnel » qui est au cœur de ses opéras s'enracine la plupart du temps dans un imaginaire musical. La conscience aiguë que le compositeur a du lien entre la musique et l'inconscient est-elle pour autant synonyme d'acquiescement aux forces primitives qui animent l'un et l'autre? La réponse, si l'on en croit Bekker, le 
spécialiste officiel de Schreker, est clairement oui. Sa lecture du conte musical de Schreker est on ne peut plus " postnietzschéenne »:

La princesse se trompe quand elle se donne au fils trop faible du maître [...] C'est dans cette erreur provoquée par les mouvements de l'âme aux interactions si complexes - appelons-les intellect, ambition ou pitié - que se situe la faute, et non pas dans l'obéissance à la pulsion. Celle-ci finit par surmonter toutes les inhibitions, tous les préjugés, toutes les hésitations. Il n'y a pas de mission plus élevée que de lui obéir, que d'aspirer à l'union avec l'être unique et complémentaire que le destin a désigné. De cette union peut naître le monde nouveau, le son lointain est proche, le carillon retentit, la mélodie du désir amoureux résonne dans toute son enivrante plénitude, le miracle de la création a lieu, la pulsion s'accomplit. [...] La mort n'est pas expiation de la faute, mais accomplissement, la pulsion n'est pas un péché, mais la loi suprême, imposée par la nature ${ }^{13}$.

Le message est clair : le vrai courage, du reste réservé aux élus, consiste à vivre ses pulsions jusqu'au bout, à être suprêmement égoïste, même si c'est au prix de la ruine et de la destruction. Dans ces lignes, le musicologue n'évoque certes pas directement le rôle joué par la musique dans ce processus. Mais on ne peut oublier la fonction essentielle du carillon : c'est par lui que se révèle au garçon, à la princesse ainsi qu'à l'ensemble de la collectivité la profondeur des pulsions qui vivent en eux. La musique donne forme retentissante à la vibration originelle, elle fait résonner, audible pour tous, ce qui, sans elle, serait resté latent ou « refoulé » au fond d'eux-mêmes. Schreker aurait ainsi écrit l'allégorie du pouvoir libérateur, désinhibateur et de facto anticivilisateur de la musique, pouvoir qu'il aurait lui-même le premier reconnu et mis au service de sa propre weltanschauung néo-nietzschéenne.

Les propos assez extrêmes de Bekker, qui voyait dans Schreker le seul digne descendant de Wagner et l'espoir d'une régénération du théâtre musical allemand, sont à replacer dans le contexte intellectuel de l'époque. En eux se reflètent une sorte de lassitude faisant suite à l'idéalisation forcenée des valeurs culturelles pratiquée au tournant du siècle, comme nous l'avons vu, tant par les héritiers du romantisme que par les adeptes de l'historicisme, et le désir de " rafraîchir » ces mêmes valeurs au contact du réel et de la vie pulsionnelle. De là à l'ennui d'une classe bourgeoise désireuse de s'offrir des émotions fortes et collectives dans un paysage intellectuel jugé trop raffiné, il n'y a qu'un pas, que Bekker franchit plus d'une fois dans son traité sur Schreker. (C'est un trait qu'on retrouve chez nombre d'intellectuels allemands de l'époque, et dont le Demian de Hesse nous donne une parfaite illustration.)

Schreker, même s'il adhère souvent aux thèses de Bekker, comme cela ressort de leur correspondance, se montre bien plus nuancé à l'intérieur de son œuvre : il montre, en réalité, à la fois les dangers d'une civilisation trop sûre d'elle-même, trop imbue de son idéalisme raffiné et ceux qu'implique une libération immodérée des pulsions. La musique du carillon, comme la sienne propre, n'est que l'expression d'un nouveau stade de conscience atteint au début du siècle : avec Schreker, l'art des sons découvre, dans le sillage de la psychanalyse, son lien avec l'inconscient et - il faut bien le reconnaître - s'enivre de cette découverte; mais celle-ci n'implique pas pour autant une soumission aveugle au diktat de la pulsion. Il convient, pour s'en convaincre, de rappeler un des axiomes principaux de la psychanalyse : on ne combat vraiment les problèmes que lorsqu'on en prend conscience. Le Carillon et la princesse montre aussi le danger de vouloir nier les forces de l'inconscient, de faire comme si la civilisation avait à jamais vaincu la pulsion de mort ou de régression. Florian a commis l'erreur de ne voir dans les aspirations les plus hautes de l'homme que l'expression d'une conscience 
et d'une volonté éthiques, oubliant qu'il y a encore là de la libido, voire du désir sexuel, à l'œuvre. Cette méconnaissance de la psyché humaine l'amène à avoir un comportement implacable, impitoyable, qui contredit son propre idéal humaniste. Non seulement il rejette son fils, mais il refuse de lui venir en aide au nom de principes quasi eugénistes: "Laisse périr, pauvre femme, ce qui n'est pas fait pour la vie ${ }^{14}$.", lance-t-il à Liese au début du premier acte. Florian reconnaîtra son erreur au deuxième acte : quand il déclare que les chants printaniers « veulent aussi connaître leur été ", il évoque de façon métaphorique la dynamique propre de l'énergie libidinale. La nostalgie [Sehnsucht] exprimée par le violon de son fils n'était qu'extérieurement dirigée vers l'idéal esthétique ; sa véritable nature était d'ordre érotique. Et le carillon n'a fait que traduire cette réalité en sons.

Bien sûr, le fait de révéler une réalité cachée ne signifie pas que cette réalité soit bonne et l'on objectera que les effets du carillon, tels qu'ils sont dépeints dans l'opéra, sont plutôt de nature dévastatrice. Pourtant, si l'on regarde de plus près la fin de l'œuvre, où se concentre l'essentiel du message ou de la morale de ce conte musical, il semble bien que la libération des pulsions reste in fine sous la domination de la culture. L'accomplissement du garçon et de la princesse, d'une part, a beau être mortifère, il n'est pas subi, mais recherché et librement consenti par les deux protagonistes. Entre eux règne une profonde affinité élective. Tous deux ont notamment le goût de l'absolu. Leur première et dernière nuit d'amour s'enveloppe de poésie et de mystère, amenant, comme dans le Tristan de Wagner, une sorte de rédemption esthétique de la pulsion de mort. D'autre part, l'ultime extase collective provoquée par le carillon s'avère quelque peu tempérée par l'intervention d'Apollon : la foule ne se livre pas, comme sept ans auparavant, à une bacchanale, mais à une ronde apaisée, image de la pulsion sublimée par l'art. Mais l'élément de réponse le plus décisif est fourni par le tableau final qui apparaît comme une variante fin de siècle de celui qui conclut le Crépuscule des dieux. Le feu consume la maison de maître Florian, tandis qu'à l'arrière-plan s'éteignent les lumières du château où s'accomplit le Liebestod de la princesse et du garçon. À ce moment là, Wolf, le principe négatif et régressif, ayant compris que son désir ne se réaliserait pas, "lance un regard vers le château baigné d'une clarté effrayante et tombe sur le sol en poussant un hurlement ${ }^{15}$ ", ce qui fait penser aux ultimes paroles et geste de Hagen, se jetant dans le Rhin dans l'espoir de récupérer l'anneau. La foule, enfin, reste sur scène, comme chez Wagner, immobile et comme abasourdie par ce qu'elle vient de vivre sous l'emprise du carillon, à présent détruit. C'est à elle qu'il revient de conclure l'opéra sur ces paroles en forme de point d'interrogation :

O Herr, wir wissen nicht aus, noch ein -

Sei uns gnädig in unseren Sünden.

O Seigneur, nous ne savons plus quoi penser -

Aie pitié de nous dans nos péchés.

Après avoir montré le pouvoir du carillon, autrement dit la capacité de la musique à exprimer et à transmettre les énergies latentes de la psyché, Schreker laisse à la foule et au public de la salle le soin de tirer eux-mêmes les conclusions de ce à quoi ils viennent d'assister ${ }^{16}$. Cette ambiguïté flottait déjà sur la fin du Crépuscule des dieux, Wagner laissant aux auditeurs le soin d'imaginer ce que pourrait être le monde d'après la catastrophe. La grande différence, c'est que l'incertitude est appelée, chez Schreker, à devenir la condition même de l'homme moderne. La musique a révélé l'étendue, la force et la complexité des pulsions à l'œuvre dans la psyché humaine et il faudra à 
présent «composer avec» cette réalité pour pouvoir envisager l'avenir de la culture avec un minimum de lucidité.

Le paradoxe qui frappe cette musique est, en définitive, historique et frappe en même temps la psychanalyse : en se plongeant dans l'inconscient elle en vient, il est vrai, à reconnaître l'étendue de son pouvoir; mais le reconnaître, c'est aussi l'affaiblir. Dans les deux cas, c'est la culture (Apollon) et non la pulsion (Dinoysos) qui a le dernier mot.

\section{NOTES}

1. Exceptés ceux de Flammen et du Schmied von Gent [Le Forgeron de Gand], respectivement le premier et le dernier opéra de Schreker.

2. Hermann Broch, Hofmannsthal und seine Zeit, in Gotthart Wunberg, Die Wiener Moderne, Reclam, Stuttgart, 1981, p. 90: "In Erfüllung seiner Traditionspflicht verwechselte Wien Museumhaftigkeit mit Kultur und wurde [...] zum Museum seiner selbst. Weil wundersamerweise Haydn und Mozart, Beethoven und Schubert sich auf diesem Erdenfleck zusammengefunden hatten, hier schlecht behandelt wurden und nichtsdestoweniger komponiert haben, richtete sich Wien als musikalische Institution ein. »

3. Notamment les Études sur l'Hystérie de Freud/Breuer, parues en 1895.

4. Traduction accompagnant l'enregistrement de l'opéra par Ulrich Windfuhr à la tête de l'orchestre philharmonique de Kiel, CD CPO, 999 958-2. L'auteur du présent article a procédé à quelques ajouts figurant entre crochets.

5. Haidy Schreker-Bures, Hören - denken - fühlen, eine kleine Studie über Schrekers Operntexte, Rimbaud Presse, Aachen, 1983, p. 24 : « Klänge, welch arg mißbrauchtes, vielgeschmähtes Wort, nur Klang, nur Klänge. Wüßten die Nörgler, welche Ausdrucksmöglichkeiten, welch unerhörter Stimmzauber ein Klang, ein Akkord in sich bergen kann. Schon als Knabe liebte ich es, mir einen jener Wagnerschen Akkorde am Klavier anzuschlagen und lauschte versunken seinem Verhallen. Wundersame Visionen wurden mir da, glühende Bilder aus musikalischen Zauberreichen und eine starke Sehnsucht. Dieser reine Klang ohne motivische Beigabe ist, mit Vorsicht gebraucht, eines der wesentlichsten musikalischen Ausdrucksmittel. »

6. «Die Natur ist eine Aeolsharfe, ein musikalisches Instrument - dessen Töne wieder Tasten höherer Sayten in uns sind. » Novalis, Das Allgemeine Brouillon, Vierte Gruppe, Nr 966, cité par Ulrike Kienzle : Das Trauma hinter dem Traum, Franz Schrekers Oper «Der ferne Klang» und die Wiener Moderne, Édition Argus, Schliengen, 1998, p. 76.

7. Jean Paul, Werke, vol. 1, München, 1980, p. 680 : «Da fing die Äolsharfe der Schöpfung an zu zittern und zu klingen, von oben herunter angeweht, und meine unsterbliche Seele war eine Saite auf dieser Laute."

8. Franz Schreker, Das Spielwerk und die Prinzessin, préface de la partition, Universal-Edition, Wien und Leipzig : «Der Meister » oder « Gott » oder die « Natur », haben in des Menschen Brust etwas Geheimnisvolles gelegt, etwas seltsam Vibrierendes, gleichsam Tönendes, das in geisternden Harmonien sich offenbart, wenn es von der Melodie irgendeiner Sehnsucht geweckt wird. Und nicht nur in des Menschen Brust schlummert, des Weckers harrend, aus feinen Membranen, Saiten, Glöckchen und Pfeifen gebildet, «das Spielwerk des Meisters Florian»; tief in der Erde Schoß pocht und bebt es und sehnt sich nach des Frühlings lockendem Ruf, nach des Sommers, der Sonne heißer Melodie. [...] Nennt es den « Trieb», den der Meister in alles, was Lebens sich 
freut, gelegt [...], das Schöpfungswunder in ewiger Erneuerung - oder nennt es « Sinnlichkeit », «verderblich Brunst» - der Ursprung ist es von allem Guten und Bösen, von Seligkeit und Enttäuschung, Glück und Elend, Leben und Tod : das Göttliche und das Teuflische.

9. Paul Bekker, Studie zur Kritik der modernen Oper, Rimbaud Presse, Aachen, 1983, pp. 26-27 : « Der ferne Klang, das Spielwerk, die Gezeichneten sind nicht drei willkürlich aneinandergereihte Bücher, der Autor greift nicht auf gut Glück bald nach diesem, bald nach jenem Stoff. Sie sind drei dramatische Phantasien über das nämliche Thema, und dieses heißt Klang. Er symbolisiert sich als geisterhafter Weckruf, als Schönheit und unheilsbringender Lebenstrieb, als verführerische, berauschende, Seelen um- und neuschaffende Sinnesmacht. In allen drei Gestaltungen ist es immer dieses eine Thema, diese eine schöpferische Entzückung des klanglichen Wundererlebnisses, der berauschenden sphärischen Geistererscheinung des Tönens. Sie ruft Schrekers dramatische Gesichte ans Tageslicht. So sehen wir hier eine Dramatik aus einer primären musikalischen Vision entwachsen, und zwar bezeichnenderweise aus dem Phänomen des neblig zerfließenden Klanges - des eigentlich transzendentalen Elementes der Musik. »

10. Franz Schreker, « Meine musikdramatische Idee » in Musikblätter des Anbruchs 1 (1919), p. 6 : «Ich schreibe planlos. Was mir einfällt ist da. Nur - ich komme von der Musik her. Meine Einfälle haben wenig « Literarisches ». Geheimnisvoll-Seelisches ringt nach musikalischem Ausdruck. Um dieses rankt sich eine äußere Handlung, die unwillkürlich schon in ihrer Entstehung musikalische Form und Gliederung in sich trägt. »

11. L'avantage décisif de la musique sur les autres arts est, de ce point de vue, tout simplement le temps, qui lui permet non seulement de symboliser mais aussi d'incarner, chaque fois qu'elle retentit, la dynamique même du désir, comme Schopenhauer l'a bien montré.

12. F. Schreker, Vorbemerkung des Autors zu dem Märchen von «Der Prinzessin und dem Spielwerk», 1913: "Denn eine Oper zu schreiben, die die Musik als Aufputz und Beiwerk erfordert, ihr nichts Selbständiges mehr zu tun übrig läßt, würde mir widerstreben; und ein Sujet $\mathrm{zu}$ vertonen, in welchem der Grundgedanke nicht ein absolut die Musik erheischender nein - die Musik selber ist, erst recht. »

13. Paul Bekker, op. cit., p. 34-35: «Die Prinzessin irrt, da sie sich dem schwachen Sohn des Meisters gibt. [...] In diesem Irren, hervorgerufen durch die sich so vielfältig überschneidenden bewegenden Mächte des Innern - nennen wir sie Intellekt, Ehrgeiz, Mitleid - liegt die Schuld, nicht aber in der Befolgung des Triebes. Dieser überwindet schließlich jede Hemmung, jedes Vorurteil, jede Zurückhaltung. Es gibt nichts Höheres als ihm zu folgen, nach der Vereinigung mit dem einen, schicksalsbestimmten, ergänzenden Wesen zu streben. In dieser Vereinigung ersteht die neue Welt, der ferne Klang ist nahe, das Spielwerk ertönt, die Melodie der Liebessehnsucht klingt in berauschender, sättigender Fülle, das Schöpfungswunder vollzieht, der Trieb erfüllt sich. [...] Der Tod ist nicht Sühnung der Schuld, sondern Erfüllung, der Trieb ist nicht Sünde, sondern oberstes Gesetz, gegeben durch Naturgebot. »

14. «Laß verkommen, Weib, was zum Leben nicht taugt. »

15. «[Wolf] blickt auf das schauerlich leuchtende Schloß und stürzt mit einem gellenden Schrei zu Boden. »

16. Dans la version remaniée de l'opéra, portant désormais le titre Das Spielwerk [Le Carillon], Schreker propose une variante plus positive du finale qui dépeint la réconciliation de Florian et de sa femme autour du cadavre de leur fils. Ce qui accrédite un peu plus l'idée que la culture humaniste reprend ses droits après les révélations du carillon. 


\section{RÉSUMÉS}

Après Le Son lointain, Schreker met à nouveau dans Le Carillon et la Princesse la musique au centre de ses préoccupations. Contrairement à son prédécesseur, le nouvel opéra de Schreker ne s'intéresse cependant plus au compositeur en quête d'inspiration mais à la musique elle-même et à son pouvoir de fascination. Toute la problématique de l'œuvre s'articule autour de l'étrange carillon de maître Florian. Cet instrument, dont les résonances magiques sont censées élever l'âme vers la spiritualité contemplative, produit en réalité l'effet inverse en entraînant son auditoire dans la sensualité et la débauche.

Sous son symbolisme parfois déconcertant, le conte musical de Schreker dissimule une réflexion particulièrement audacieuse sur le lien entre la musique et l'inconscient. Bon connaisseur des premiers écrits psychanalytiques, le compositeur viennois prolonge et approfondit les théories musicales de Schopenhauer en mettant en lumière la correspondance à la fois physique et symbolique qui relie le son à la pulsion. Il ouvre ce faisant un questionnement toujours actuel sur la place qu'il convient d'accorder au sein de la civilisation, et de l'art en particulier, à la libération des pulsions.

Nach dem Fernen Klang stellt Schreker in seiner Oper Das Spielwerk und die Prinzessin erneut die Musik in den Mittelpunkt seines Schaffens. Nicht mehr aber für den nach Inspiration suchenden Komponisten, sondern für die Musik selbst und deren Zauberkraft interessiert sich nunmehr Schreker. Die ganze Problematik des Werkes dreht sich um das wundersame Instrument Meister Florians. Dieses Spielwerk, dessen Resonanzen ursprünglich die Seele der Zuhörer in einen Zustand geistiger, kontemplativer Seligkeit versetzen sollten, liefert umgekehrt sein Publikum der alles bezwingenden Macht der Sinnlichkeit aus.

Unter seiner oft verwirrenden Symbolik verbirgt das musikalische Märchen Schrekers eine besonders moderne Reflexion über das Verhältnis der Musik zum Unbewußten. Als guter Kenner der frühen psychoanalytischen Schriften, ergänzt und vertieft der Wiener Komponist die Schopenhauerschen Musiktheorien, indem er in seiner Oper auf die zugleich physischen und symbolischen Entsprechungen zwischen Klang und Trieb hinweist. Dabei wirft er die stets aktuelle Frage auf, welchen Platz die Entfesselung der Triebe innerhalb der Kultur bzw. der Kunst beanspruchen kann.

\section{AUTEUR}

\section{ALAIN LEDUC}

Université du Littoral - Côte d'Opale, Boulogne-sur-Mer 